INPLASY

PROTOCOL

To cite: Rodolico et al. Efficacy and safety of ketamine for unipolar and bipolar depression: an overview of reviews. Inplasy protocol 202150049. doi:

10.37766/inplasy2021.5.0049

Received: 13 May 2021

Published: 13 May 2021

Corresponding author:

Alessandro Rodolico

alessandro.rodolico@phd.unict.it

Author Affiliation:

Department of Clinical and Experimental Medicine, Psychiatry Unit, University of Catania, Catania, Italy.

Support: None.

Review Stage at time of this submission: Preliminary searches.

Conflicts of interest: None declared.

\section{Efficacy and safety of ketamine for unipolar and bipolar depression: an overview of reviews}

Rodolico, A1; Natale, A2; Rapisarda, L33; Concerto, C4; Francesco, AD5; Petralia, A6; Aguglia, E7.

Review question / Objective: This review aims to summarize evidence from systematic reviews with meta-analyses on the efficacy of monotherapy or add on administration ketamine (all available formulations) in reducing suicide risk, depressive symptoms, response to treatment, remission, relapse rate, dropout rate and side effects (dissociation, psychosis), compared to placebo or any other drug for unipolar and bipolar depression. We plan to assess the quality of the evidence for key outcomes and to identify improvements needed in future research.

Condition being studied: Major depressive disorder (with or without treatment resistance) and Bipolar disorder (with or without treatment resistance).

INPLASY registration number: This protocol was registered with the International Platform of Registered Systematic Review and Meta-Analysis Protocols (INPLASY) on 13 May 2021 and was last updated on 13 May 2021 (registration number INPLASY202150049).

\section{INTRODUCTION}

Review question / Objective: This review aims to summarize evidence from systematic reviews with meta-analyses on the efficacy of monotherapy or add on administration ketamine (all available formulations) in reducing suicide risk, depressive symptoms, response to treatment, remission, relapse rate, dropout rate and side effects (dissociation, psychosis), compared to placebo or any 
other drug for unipolar and bipolar depression. We plan to assess the quality of the evidence for key outcomes and to identify improvements needed in future research.

Rationale: In the last years several studies suggested an important antidepressant effect of ketamine, an aryl-cyclo-alkylamine drug, to treat mood disorders. On the 5th of March, 2019 FDA approved the use of esketamine for intranasal administration combined with another oral antidepressant for the treatment of Major Depressive Disorder (MDD). The interest on this new drug led to an increase of randomized controlled trials (RCTs), systematic reviews and meta-analyses. An excess of information not always contribute effectively to the research area if the available data are not qualitatively processed. Thereafter, the main scope of this project is meta-summarizing the available evidence synthesis works and evaluate their overall quality.

Condition being studied: Major depressive disorder (with or without treatment resistance) and Bipolar disorder (with or without treatment resistance).

\section{METHODS}

Search strategy: A comprehensive literature searches of reviews up to March 2021 was performed using PubMed/ MEDLINE, Cochrane Library, Embase. The search strategy included words relating to the terms: 'ketamine', 'n-methylketamine', 'n methylketamine' and 's ketamine'. We focused our search on systematic reviews and meta-analyses using the appropriate filters of each scientific search engine.

Participant or population: Included: Adults (from 18 to 65 years old), affected by Major Depressive Disorder or Bipolar Disorder (with or without treatment resistance). Excluded: Comorbidities with substance use disorder or with personality disorder.

Intervention: Ketamine in monotherapy or add-on administration to other drugs, independently from route of administration.
Comparator: Placebo or other drugs.

Study designs to be included: Systematic reviews with meta-analyses of randomized controlled trials.

Eligibility criteria: All the following criteria have to be satisfied for inclusion: Systematic reviews (with meta-analysis): 1) On participants affected by affective disorders (bipolar and depressive disorder with or without treatment resistance). Animal and in vitro models will be excluded. 2) On Ketamine (all available formulations) in monotherapy or add on administration. 3) Where the comparator is placebo or any other antidepressant. 4) Where at least one efficacy outcome (suicide risk or depressive symptoms or response to treatment or remission or relapse rate or dropout rate) or safety measures (dissociation or psychosis) is reported. We will exclude systematic reviews that focus only on Ketamine as pre-ECT anaesthetic adjuvant, but we will include reviews that present the outcomes separately for trials on Ketamine alone and Ketamine as pre-ECT anaesthetic adjuvant, considering only the former ones.

Information sources: Electronic databases were undertaken using: Embase; Medline (access via PubMed) and Cochrane Library. Contact with authors.

Main outcome(s): Efficacy in reducing suicide risk.

Additional outcome(s): Efficacy in reducing depressive symptoms, response to treatment, remission, relapse rate, dropout rate and side effects (dissociation, psychosis).

Data management: Data will be extracted in a relational database by two independent reviewers who also will estimate the overall quality of the Systematic Reviews. If any discrepancy is present in extracted data, the reviewers will reach a final agreement. If the two reviewers will not be able to resolve the disagreement a third reviewer will intervene in the final decision. 
Quality assessment / Risk of bias analysis: The quality of systematic reviews for the key outcomes will be assessed using the AMSTAR 2 checklist. It will be reported, if available, the confidence on outcomes derived from GRADE scores of the systematic reviews.

Strategy of data synthesis: Structured descriptive tables will be used to summarize the characteristics of the studies and the quality of the evidence of the included reviews. The effect sizes from the meta-analyses will be presented as mean differences (WMD), standardized mean differences (SMD), odds ratios (OR), relative risks (RR) or risk differences (RD), depending on the data reported by the authors. In addition, whenever possible, the results will be reported with $95 \%$ confidence intervals $(95 \% \mathrm{Cl})$. It is not planned to conduct any further metaanalysis on the data.

Subgroup analysis: No sub-group metaanalyses are planned. We will present the summery tables divided by diagnosis and outcome.

Sensitivity analysis: Not applicable.

Language: English.

Country(ies) involved: Italy.

Keywords: systematic review; ketamine; reviews; meta analysis; overview; mood disorder; major depressive disorder; bipolar disorder; suicide.

Dissemination plans: We plan to publish the present work in peer reviewed journals and present it in scientific conferences.

Contributions of each author:

Author 1 - Alessandro Rodolico.

Email: alessandro.rodolico@phd.unict.it

Author 2 - Antimo Natale.

Email: antimo.natale@studium.unict.it

Author 3 - Laura Rapisarda.

Email: rapisarda.laura@studium.unict.it

Author 4 - Carmen Concerto.

Email: carmenconcerto@hotmail.it
Author 5 - Antonio Di Francesco.

Email: antonio.difrancesco@studium.unict.it

Author 6 - Antonino Petralia.

Email: petralia@unict.it

Author 7 - Eugenio Aguglia.

Email: eugenio.aguglia@unict.it 